\title{
Examination stress and components of working memory
}

\author{
RICHARD S. LEWIS ${ }^{1}$, ANI NIKOLOVA ${ }^{2}$, DENNIS J. CHANG ${ }^{1}$, \& NICOLE Y. WEEKES ${ }^{1}$ \\ ${ }^{1}$ Department of Psychology and Neuroscience Program, Pomona College, Claremont, CA, USA, and ${ }^{2}$ Department of \\ Psychology, Claremont Graduate University, Claremont, CA, USA
}

(Received 30 March 2007; revised 21 May 2007; accepted 22 fune 2007)

\begin{abstract}
Previous research suggests that stress can influence a broad range of memory functions. In this study we investigated the effect of a naturalistic stressor, examination stress, on working memory in young adults. In order to accomplish this aim, participants were tested on psychological and hormonal measures of stress and on Digit Span, once during a low stress period and once during a high stress period. The high examination stress condition was associated with an increase in cortisol and subjective impressions of stress. Although Digits Forward performance did not vary with examination stress, Digits Backward performance improved. These findings suggest that mild increases in stress are associated with improvement in the manipulation component of working memory. However, no correlations were found between working memory and either cortisol or psychological stress. Thus the mechanism by which mild naturalistic stressors improve the manipulation component of working memory needs further investigation.
\end{abstract}

Keywords: Cortisol, Digit Span, executive control, inverted U-curve, stress, working memory

While most of the studies on stress and memory have focused on the impact of elevations of glucocorticoids on long-term declarative memory, relatively few studies have investigated the role of hormonal elevations on working memory. Yet there is some evidence to suggest that acute increases in cortisol may have a more dramatic effect on working memory than on long-term, declarative memory (Lupien and Lepage 2001).

In general, three approaches have been taken to studying stress and working memory in humans. The first approach uses hydrocortisone administration. Generally these studies have found deficits in working memory (Lupien et al. 1999; Young et al. 1999; Wolf et al. 2001), although a few studies did not find any effects on Digit Span (Lupien et al. 1999; Kuhlmann et al. 2005), a frequently used clinical and experimental measure of working memory. A strength of the hydrocortisone administration approach is the control it provides through directly increasing glucocorticoid level in relative isolation from other hormones. One limitation, however, is that the approach has only limited ecological validity. For example, the elevations in cortisol that result from hydrocortisone administration have been substantially higher than those occurring naturally, ranging from a $200 \%$ increase in urinary cortisol (Young et al. 1999) to an $800 \%$ increase in salivary cortisol (Kuhlmann et al. 2005). Furthermore, increases in cortisol induced by hydrocortisone may not be associated with increases in subjective accounts of stress (Wachtel et al. 2001), which typically result following naturally occurring stressors (Smyth et al. 1998).

A second approach has been to induce stress in the laboratory through use of public speaking and demanding arithmetic tasks, such as occurs in the Trier Social Stress Test (TSST) (Kirschbaum et al. 1996). While some findings have suggested no effect of such laboratory stressors on Digit Span (Kuhlmann et al. 2005) or impairment in only Digits Forward of cortisol responders (Elzinga and Roelofs 2005), other findings have suggested an increase in the storage

Correspondence: R. S. Lewis, Department of Psychology and Neuroscience Program, Pomona College, 647 N. College Way, Claremont, CA 91711, USA. Tel: 1909607 2445. Fax: 1909621 8623. E-mail: rlewis@pomona.edu 
component and a decrement in the manipulation component of working memory (al'Absi et al. 2002). One advantage of laboratory stressors, like the TSST, is that it can be administered under controlled, laboratory conditions. It also has greater ecological validity than hydrocortisone administration due to its modeling of naturally-occurring stressors. Moreover, laboratory stressors show more modest increases in cortisol than do hydrocortisone administration studies, ranging from a 50\% increase (Elzinga and Roelofs 2005) to a 100\% increase (al'Absi et al. 2002). However, it is still not clear to what extent laboratory stressors may be externally valid and generalize to naturally occurring settings (Lundberg et al. 1990; Spangler 1997; Cohen et al. 2000).

A third approach, which has received the least attention, has been to measure working memory in response to naturalistic stressors. A major strength of the naturalistic stressor approach is its external validity. However, this approach may also have limited internal validity. For instance, Vedhara et al. (2000) tested students during a non-examination period and during an examination period and found an increase in subjective stress during the examination period, but a decrease in salivary cortisol. No change was found in working memory performance between the nonexamination and examination conditions. Klein and Boals (2001) found that reports of stressful life events were correlated with a decrease in working memory; however, no measure of cortisol was taken in their study. Because of these inconsistent findings and the paucity of relevant studies, the relationship among psychological stress, cortisol, and working memory in naturalistic situations remains unclear.

An additional issue in this literature is that even when alterations in working memory are found, it is not always clear which stage of working memory is most affected. Working memory refers to the temporary storage, maintenance, and manipulation of information and involves several dissociable components. Mechanisms for the storage of information have been associated with posterior brain structures, whereas mechanisms for manipulation of information, or executive control, have been associated with anterior brain structures (D'Esposito and Postle 2002). Of the studies that measured both storage and executive control, the results have been inconclusive. For example, Wolf et al. (2001) found a decrement in storage and executive control, Lupien et al. (1999) found no change in storage but a decrease in executive control, and Elzinga and Roelofs (2005) found impairment only in storage.

The primary goal of the present study was to investigate how a naturalistic stressor, examination stress, affects the storage and executive control elements of working memory using Digit Span. We measured working memory using Digit Span for two reasons. First, it is the single most widely used task in the stress and working memory literature, as well as one of the most widely used measures of working memory in clinical and experimental studies (Lezak 1995). Second, Digit Span can also be used to assess different components of working memory; Digits Forward being associated with storage and Digits Backward being associated with executive control (D'Esposito and Postle 1999, 2002). Furthermore, because recent data have questioned the coincidence of psychological and physiological measures of stress (Dickerson and Kemeny 2004), we measured the subjects' perception of stress as well as salivary cortisol, a hormonal index of stress. Finally, we used a naturalistic stressor, examination stress, in order to increase the generalizability of our findings.

\section{Method}

\section{Participants}

Sixty-seven undergraduates, ages 18-22 years (33 males and 34 females) served as participants. The investigation of the relationship between stress and working memory was part of a larger study on stress and brain activity (Weekes et al. 2006). Twentyseven participants were tested during the summer and fall of 2003 (Group A) and 40 participants were tested during the spring and summer of 2004 (Group B). Exclusion criteria included: (i) smokers, (ii) lefthanders (excluded because of concern about reversed language dominance during the event-related potential (ERP) memory task, not included in this report), (iii) non-native English speakers, (iv) those with vision that was not corrected to normal, (v) antihistamine, glucocorticoid or asthma medication users, (vi) those with exposure to general anesthesia in the last year, (vii) those with a personal or first degree family diagnosis of a DSM-IV, Axis I disorder, and (viii) those with endocrine abnormalities.

\section{Psychological materials}

Spielberger State Trait Anxiety Inventory (STAI; Spielberger 1983)

The State Inventory. This 20-item scale is the subscale of the Spielberger State Trait Anxiety Inventory (STAI-S) that assesses anxiety at the time of testing. The Spielberger state anxiety inventory questions how participants "feel right now" regarding items such as, "I wish I could be as happy as others seem to be" and "I have disturbing thoughts" rated on a 4-point scale in which 1 signifies "almost never" and 4 signifies "almost always."

The trait inventory. This 20 -item scale is the subscale of the Spielberger State Trait Anxiety Inventory (STAIT) that assesses chronic anxiety. The Spielberger Trait Anxiety Inventory questions participants about how they "feel generally" regarding items such as, 
"I wish I could be as happy as others seem to be" and "I have disturbing thoughts" rated on a 4-point scale in which 1 signifies "almost never" and 4 signifies "almost always."

Perceived Stress Scale (PSS; Cohen et al. 1983). This 14 -item inventory assesses the frequency of feelings of anxiety regarding certain potentially stressful events over the preceding month. The 5-point scale includes items such as "In the last month, how often have you been angered because of things that happened that were outside of your control?" and "In the last month, how often have you felt difficulties were piling up so high that you could not overcome them?" Responses range from 0 indicating "never" to 4 indicating "very much so.”

Digit Span. The Digit Span subtests were derived from the Wechsler Memory Scale-Revised (WMS-R) (Wechsler 1987) and the Wechsler Adult Intelligence Scale-Revised (WAIS-R) (Wechsler 1981). In order to make the two versions of Digit Span comparable, the last trial of the Digits Forward and Digits Backward subtests from the WAIS-R were eliminated. Therefore, the Digits Forward subtest consisted of Trial I and Trial II for six items ranging from three digits to eight digits. The Digits Backward subtest consisted of Trial I and Trial II for six items ranging from two digits to seven digits. The maximum number of digits in the final sequences was the same as in other studies using Digit Span to investigate the effects of stress on working memory (Wolf et al. 2001; Elzinga and Roelofs 2005; Kuhlmann et al. 2005). Digit Span was administered and scored according to the Wechsler manuals. Digits were presented one per second and participants were required to repeat the digits in order for Digits Forward and in reversed order for Digits Backward. Each correct trial received a score of one point. There were a total of 12 possible points for each subtest. Testing was discontinued if the subject failed at both trials within a given digit series length.

\section{Design}

A counterbalanced, within-participants design was implemented in this study where examination stress (low examination period, high examination period) was the one consistent within-participants factor. There were two counterbalanced groups of participants, Group A was tested during summer and fall of 2003 and Group B was tested during spring and summer of 2004. Sex of subject served as the second between-subjects factor. The dependent variables measured were level of psychological stress (i.e. state anxiety, trait anxiety, and perceived stress), hormonal stress (i.e. salivary cortisol level), and performance on Digits Forward and Digits Backward.

\section{Procedure}

All participants came into the laboratory individually, once during a low examination stress period, and once during a high examination stress period. All low examination stress testing occurred during either Summer 2003 or Summer 2004, when no classes were in session. All high examination stress testing occurred during either Fall 2003 or Spring 2004. In order to avoid the sharp decline in cortisol observed during the hours following morning awakening (see Kirschbaum and Hellhammer 1994 for a review of this issue), all subjects were tested in the afternoon or early evening hours. A typical session started at either $3: 30 \mathrm{pm}$ or 5:30 pm For any one individual, there was no more than a $1 \mathrm{~h}$ discrepancy between the commencement of the low stress and high stress testing sessions. The two sessions occurred approximately three months apart. Sessions were approximately $2 \mathrm{~h}$ in length.

The two sessions differed only in whether the session was scheduled during a time of high or low examination stress. The high examination session was defined as a session when the subject had at least three major examinations or assignments due that week. A low examination session was defined as a session during the summer when no classes were in session and when the subject had no major examination or assignments due.

After providing informed consent, participants completed a packet of stress inventories and were given one salivette to provide one salivary sample. Subjects then participated in an ERP encoding task (not described here). After approximately $30 \mathrm{~min}$ of the ERP encoding task, participants were administered the Digit Span Test. This was followed by $40 \mathrm{~min}$ of an ERP recognition task. At the end of testing, participants were given a second salivette and provided a second salivary sample. Samples were placed in a $-20^{\circ} \mathrm{C}$ freezer. After all samples had been collected, they were sent to Salimetrics, Inc. in State College, PA to be analyzed for cortisol via enzymelinked immunosorbent assay (ELISA). The average concentration of cortisol in the two salivary samples taken at each session was used as a measure of physiological stress. The study protocol was approved by the Pomona College Institutional Review Board for Human Subject Protection.

\section{Results}

Ten of the original 67 subjects were excluded because of their extreme scores based on an outlier analysis of cortisol samples. This is consistent with the approach that we have taken for subject exclusion in our previous work on this sample population (Weekes et al. 2006). More specifically, of the 10 subjects who were excluded, all had cortisol concentrations that were at least $2.5 \mathrm{SDs}$ above the mean. Of the remaining 
57 subjects, one subject had a Digits Backward value less than three standard deviations below the mean, and eight had missing data. Thirteen of the excluded subjects were male and six were female (leaving 20 males and 28 females in the final analysis). Eight of the excluded subjects were from Group A, and 10 were from Group B, leaving 18 subjects from Group A and 30 from Group B.

Analyses were performed on the remaining 48 subjects. For the working memory variables, a single omnibus $2 \times 2 \times 2 \times 2$ mixed ANOVA was performed with Counterbalancing Group (A, B) and Sex (female, male) as between-subject factors and with Session (low examination period, high examination period) and Digit Span subtest (digit forward, digit backward) as the within-subject factors. Means and standard deviations for the dependent variables for the low and high examination sessions are presented in Table I.

For each stress-related dependent variable (psychological measures of stress and cortisol concentrations), a $2 \times 2 \times 2$ mixed ANOVA was performed with Counterbalancing Group (A,B) and Sex (female, male) as between-subject factors and with Session (low examination period, high examination period) as the within-subject factor. Means and standard deviations for the dependent variables for the low and high examination sessions are presented in Table I.

\section{Working memory: Digit Span}

Performance was found to significantly vary between the low and high examination stress sessions, $F(1,44)=131.86, p<0.0001, \eta^{2}=0.75$, such that overall performance was better during the high examination stress session than during the low examination stress session. In addition, a significant interaction was found between Session and Digit Span subtest, $F(1,44)=5.27, p=0.03, \eta^{2}=0.11$, such that the Digits Forward subset showed no sessionrelated performance difference $(t(47)=0.90, \mathrm{~ns})$, whereas the Digits Backward subtest showed significantly greater performance during the high examination stress session than during the low examination stress session $(t(47)=-2.58, p=0.02)$. No other main effects or interactions were found to be significant. Of critical interest to the present investigation, no significant interactions were found between session and sex, $F(1,44)=0.24$, ns, $\eta^{2}=0.05$; session and group, $F(1,44)=2.13$, ns, $\eta^{2}=0.05$, or session by sex by group, $F(1,44)=0.63$, ns, $\eta^{2}=0.01$.

Even though the maximum number of digits used in the Digits Forward subtest was the same as used in other studies of stress and working memory, 14 of our subjects had perfect scores (12) on one of the Digits Forward measures. Therefore, we also ran separate analyses with only those 34 subjects without a perfect score. This was done in order to rule out a ceiling effect attenuating a difference on Digits Forward between the low and high examination stress sessions. For the subjects without perfect scores, Digits Forward was still not found to significantly vary between the low and high examination stress sessions, $F(1,30)=0.27$, ns, $\eta^{2}=0.009$. In addition, no interactions were found between the examination sessions and sex, $F(1,30)=0.64, \mathrm{~ns}, \eta^{2}=0.02$, session and group, $F(1,30)=0.002, \mathrm{~ns}, \eta^{2}=0.001$, nor session by sex by group, $F(1,30)=0.10$, ns, $\eta^{2}=0.003$. Reduced sleep and greater caffeine use during the high examination stress period (based on data from 40 subjects, $t(39)=2.78, p=0.008)$ showed no correlation with performance.

\section{Psychological stress}

All of the subjective measures of stress were significantly higher during the high examination stress session than during the low examination session (STAI-S: $F(1,44)=7.76, \quad p=0.008, \quad \eta^{2}=0.15 ; \quad$ STAI-T: $F(1,44)=4.96, p=0.03, \eta^{2}=0.10$; PSS: $F(1,44)=$ 9.34, $\left.p=0.004, \eta^{2}=0.18\right)$. However, no interactions were found between session and sex (STAIS: $F(1,44)=$ 1.03, ns, $\eta^{2}=0.02$; STAI-T: $F(1,44)=0.30$, ns, $\eta^{2}=0.007$; PSS: $F(1,44)=0.03$, ns, $\left.\eta^{2}=0.001\right)$, session and group (STAIS: $F(1,44)=0.31$, ns, $\eta^{2}=$ 0.007; STAI-T: $F(1,44)=0.01$, ns, $\eta^{2}=0.001$; PSS: $F(1,44)=0.16$, ns, $\left.\eta^{2}=0.004\right]$, or session by sex

Table I. Mean scores of stress measures and Digit Span (and standard deviations) as a function of examination stress.

\begin{tabular}{|c|c|c|}
\hline & Low examination stress & High examination stress \\
\hline State anxiety (STAI-S) $\star \star \star ~$ & $32.89(6.60)$ & $37.647(10.28)$ \\
\hline Trait anxiety $(\text { STAI-T) })^{\star \star}$ & $35.83(7.23)$ & $37.71(7.86)$ \\
\hline 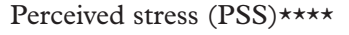 & $19.33(6.49)$ & $22.79(6.97)$ \\
\hline Salivary cortisol $(\mathrm{ug} / \mathrm{dl})^{\star}$ & $0.09(0.03)$ & $0.11(0.04)$ \\
\hline Digit forward & $10.7(1.36)$ & $10.04(1.30)$ \\
\hline Digit backward $\star \star$ & $7.79(1.83)$ & $8.52(1.72)$ \\
\hline
\end{tabular}

${ }^{\star} p=0.05 ;{ }^{\star \star} p<0.05 ;{ }^{\star \star \star} p<0.01 ;{ }^{\star \star \star \star} p<0.005$. 
by group [STAIS: $F(1,44)=1.88$, ns, $\eta^{2}=0.04$; STAI-T: $F(1,44)=0.42$, ns, $\eta^{2}=0.009$; PSS: $\left.F(1,44)=0.10, \mathrm{~ns}, \eta^{2}=0.002\right]$

\section{Cortisol}

Salivary cortisol concentration was also significantly greater during the high examination stress session than during the low examination session, $F(1,44)=4.00 ; p=0.05, \eta^{2}=0.08$. However, no interactions were found between session and sex, $F(1,44)=2.02$, ns, $\eta^{2}=0.04$, session and group, $F(1,44)=1.47$, ns, $\eta^{2}=0.03$ or session by sex by group, $F(1,44)=1.21$, ns, $\eta^{2}=0.03$.

\section{Relationship among variables}

Because there is variability in how people respond to and perceive stressful situations, we also investigated whether there was a relationship between changes in stress and working memory performance. Therefore, we conducted a correlational analysis between the percent change in stress measures across the two sessions and the percent change in Digit Span measures across the two sessions (see Table II for results). No significant correlations were observed between the stress measures and either the Digits Forward or Digits Backward subtests. We also calculated correlations among these dependent variables for individual sessions, but none of these correlations were significant either. Furthermore, we performed a curve fitting analysis to test for quadratic relationships. Similarly no significant relationships were found (see Table III for results).

Finally, the result that no significant interactions were found with either the sex or the group of the participants suggest that enhanced performance for Digits Backward during the high examination stress session was dependent neither on whether the subject was male or female, nor on whether the high examination stress session was the first (as in Group B) or the second (as in Group A) session in which individual subjects participated.

\section{Discussion}

The examination stress design used in the present study resulted in an increase in perception of stress by the subjects as well as in their salivary cortisol levels. Exposure to stress did not affect Digits Forward scores, but did increase Digits Backward scores. These findings suggest that exposure to examination stress results in an increase in the manipulation component of working memory, a component that has been linked to frontal systems involved in working memory (D'Esposito and Postle 2002).

These results are in contrast with previous naturalistic stressor studies, which either did not measure cortisol, but did find a decrease in working memory (Klein and Boals 2001), or found a decrease in cortisol, an increase in perception of stress, and no change in working memory (Vedhara et al. 2002). These results are also in contrast with the hydrocortisone administration studies, which generally found a decrement in working memory (Lupien et al. 1999; Young et al. 1999; Wolf et al. 2001), although Lupien et al. (1999) and Kuhlmann et al. (2005) did not find an effect of stress on Digit Span, the task used in our study. Furthermore, our results appear to be at odds with the laboratory stressor studies, which found no effect on Digit Span (Kuhlmann et al. 2005), a decrement in Digits Forward, which measures the storage component of working memory (Elzinga and Roelofs 2005), and a decrement in the manipulation component of working memory (al'Absi et al. 2002).

One strategy for resolving these seemingly inconsistent findings is to consider the different levels of cortisol increase that result from the three different approaches

Table II. Pearson product-moment correlations between percent change in Digit Span and percent change in stress measures across the two sessions $(n=48)$.

\begin{tabular}{lccrr}
\hline & $\begin{array}{c}\text { State anxiety } \\
\text { session change }\end{array}$ & $\begin{array}{c}\text { Trait anxiety } \\
\text { session change }\end{array}$ & $\begin{array}{c}\text { Perceived stress } \\
\text { session change }\end{array}$ & $\begin{array}{r}\text { Salivary cortisol } \\
\text { session change }\end{array}$ \\
\hline Digits Forward & 0.21 & 0.17 & 0.18 & 0.05 \\
Digits Backward & -0.24 & -0.10 & -0.16 & -0.18 \\
\hline
\end{tabular}

Note: none of the correlations are statistically significant.

Table III. Quadratic curve fitting $R$ values between percent change in Digit Span and stress measures $(n=48)$.

\begin{tabular}{lcccc}
\hline & State anxiety & Trait anxiety & Perceived stress & Salivary cortisol \\
\hline Digits Forward & 0.26 & 0.17 & 0.20 & 0.17 \\
Digits Backward & 0.24 & 0.17 & 0.14 & 0.17 \\
\hline
\end{tabular}

Note: none of the $R$ values are statistically significant. 
to stress manipulation. Specifically, the hydrocortisone administration approach leads to the highest percentage increase in cortisol level, while laboratory stressors lead to a moderate increase, and examination stressors lead to a more modest increase. Furthermore, the relationship between cortisol increase and performance may not follow a linear relationship. Indeed, numerous studies have suggested an inverted $U$ relationship between stress and memory (Lupien and McEwen 1997; Lupien and Lepage 2001). Indeed, Lupien et al. (1999) reported such an inverted U relationship between hydrocortisone dose and working memory such that impairment in working memory was found only at the highest dose of hydrocortisone. The inverted $U$ relationship between memory and stress is reminiscent of Yerkes and Dodson's (1908) seminal paper describing optimal learning occurring at moderate levels of motivation.

Additionally, the specific nature of the relationship between stress and working memory may be dependent on the component of working memory assessed. Because glucocorticoid receptors are in higher concentration in anterior cortical areas, we might expect stress to have its greatest effect on the manipulation or executive control component of working memory (D'Esposito and Postle 2002).

Therefore, we might also expect that minor increases in cortisol would be associated with facilitation of the manipulation component of working memory, as was found in our study. Large increases in cortisol, which typically are found in hydrocortisone administration studies, would be expected to be associated with decrements in working memory, as has been found in previous administration studies. Finally, laboratory stressor studies, such as the TSST, which have produced more moderate levels of cortisol increases, might be expected to show no change in working memory or as a group yield more inconsistent results, depending on the level of increase in cortisol.

The failure to find a relationship between the stress measures and working memory performance suggests that there is not a direct relationship between the increase in stress and working memory performance. Generally, studies on stress and working memory have not reported finding relationships between changes in cortisol and corresponding changes in working memory (Young et al. 1999; Vedhara et al. 2000; Wolf et al. 2001; al'Absi et al. 2002; Kuhlmann et al. 2005). However, as previously noted, Lupien et al. (2001) did find an inverted U relationship between hydrocortisone dose and working memory performance. Elzinga and Roelofs (2005) also reported a relationship between cortisol and working memory such that increases in cortisol were negatively associated with working memory performance on Digit Span. Together, these findings leave the mechanism between stress and working memory unresolved.
Certainly, students preparing for examinations may have experienced increases in functions other than stress. Moreover, such responses may facilitate their working memory performance compared to nonexamination periods. Moderate increases in arousal, motivation, and attention have all been shown to conform to the Yerkes-Dodson Law. For instance, Nielson et al. (1996) investigated the relationship between arousal and memory by varying muscle tension, a function associated with catecholamine release (Nielson and Jenson 1994). They found that moderate levels of arousal were associated with peak memory performance. While cortisol is released during prolonged and intensely stressful experiences, catecholamines are released acutely and in response to even mild or moderate stressful experiences (McEwen and Sapolsky 1995). As a mild to moderate stressor, examination stress may have effects on working memory through catecholamines. While the interactions between glucocorticoids and catecholamines on working memory have received some attention (Roozendaal et al. 2004), they are in need of further investigation and empirical support.

It is, therefore, possible that the increase in Digits Forward performance observed during the high examination stress period may have been more directly associated with increases in arousal, motivation, or attention. It is also possible that memory practice while studying for examinations may have facilitated working memory performance during the examination stress session.

In conclusion, findings from the present study suggest that stressors experienced naturally may improve the manipulation or executive control component of working memory. This effect may be due to the relatively mild increase in stress measured using the examination stress design. Together with the existing literature, these findings suggest that there are a multitude of factors that may influence the relationship between stress and working memory. Chief among these are: glucocorticoid levels, catecholamine levels, and the component of working memory measured. In addition gaining a better understanding of how these factors, in addition to arousal, attention, motivation, and memory practice, interact will be essential for understanding the role of stress on working memory.

\section{Acknowledgements}

This research was supported by a grant from the National Science Foundation (NSF-BCS0224069) awarded to N.Y. Weekes and R.S. Lewis. We acknowledge Salimetrics, LLC for performing the biological assays and thank Dale Berger, Jared Garrison-Jakel, Ambereen Kurwa, Erin Mohr, Falgooni Patel, Rebecca Sheehan-Stross, Elizabeth Volkmann, and Tracy Wang for their assistance in conducting this study. 


\section{References}

al'Absi M, Hugdahl K, Lovallo WR. 2002. Adrenocortical stress responses and altered working memory performance. Psychophysiology 39:95-99.

Cohen S, Kamarck T, Mermelstein RA. 1983. A global measure of perceived stress. J Health Soc Behav 24:385-396.

Cohen S, Hamrick N, Rodriguez MS, Feldman PJ, Rabin BS, Manuck SB. 2000. The stability of and intercorrelations among cardiovascular, immune, endocrine, and psychological reactivity. Ann Behav Med 22:171-179.

D'Esposito M, Postle BR. 1999. The dependence of span and delayed-response performance on prefrontal cortex. Neuropsychologia 37:1303-1315.

D'Esposito M, Postle BR. 2002. The neural basis of working memory storage, rehearsal, and control processes. In: Squire LR, Schacter DL, editors. Neuropsychology of memory. 3rd ed., New York: Guilford. p 215-224.

Dickerson SS, Kemeny ME. 2004. Acute stressors and cortisol responses: A theoretical integration and synthesis of laboratory research. Psychol Bull 136:355-391.

Elzinga BM, Roelofs K. 2005. Cortisol-induced impairments of working memory require acute sympathetic activation. Behav Neurosci 119(1):98-103.

Kirschbaum C, Hellhammer DH. 1994. Salivary cortisol in psychoneuroendocrinology research: Recent developments and applications. Psychoneuroendocrinology 19:313-333.

Kirschbaum C, Pirke KM, Hellhammer DH. 1996. The "trier social stress test" - a tool for investigating psychobiological stress responses in a laboratory setting. Neuropsychobiology 28:76-81.

Klein K, Boals A. 2001. The relationship of life event stress and working memory capacity. Appl Cognit Psychol 15:565-579.

Kuhlmann S, Kirschbaum C, Wolf OT. 2005. Effects of oral cortisol treatment in healthy young women on memory retrieval of negative and neutral words. Neurobiol Learn Mem 83: $158-162$.

Kuhlmann S, Piel M, Wolf OT. 2005. Impaired memory retrieval after psychosocial stress in healthy young men. J Neurosci 25: 2977-2982.

Lezak MD. 1995. Neuropsychological assessment. New York: Oxford University Press.

Lundberg U, Melin B, Fredrickson M, Tuomisto M, Frankenhaeuser M. 1990. Comparison of neuroendocrine measurements under laboratory and naturalistic conditions. Pharmacol Biochem Behav 37:697-702.

Lupien SJ, McEwen BS. 1997. The acute effects of corticosteroids on cognition: Integration of animal and human model studies. Brain Res Rev 24:1-27.

Lupien SJ, Lepage M. 2001. Stress, memory, and the hippocampus: Can't live with it, can't live without it. Behav Brain Res 127: 137-158.
Lupien SJ, Gillin CJ, Hauger RL. 1999. Working memory is more sensitive than declarative memory to the acute effects of corticosteroids: A dose-response study in humans. Behav Neurosci 113(3):420-430.

McEwen BS, Sapolsky RM. 1995. Stress and cognitive function. Curr Opin Neurobiol 5:205-216.

Nielson KA, Jensen RA. 1994. Beta-adrenergic receptor antagonist antihypertensive medications impair arousal induced modulation of working memory in elderly humans. Behav Neural Biol 62:190-200.

Nielson KA, Radtke RC, Jensen RA. 1996. Arousal-induced modulation of memory storage processes in humans. Neurobiol Learn Mem 66:133-142.

Roozendaal B, McReynolds, Jr., McGaugh JL. 2004. The basolateral amygdala interacts with the medial prefrontal cortex in regulating glucocorticoid effects on working memory impairment. J Neurosci 11:1385-1392.

Smyth J, Ockenfels M, Porter L, Kirschbaum C, Hellhammer D, Stone A. 1998. Stressors and mood measured on a momentary basis are associated with salivary cortisol secretion. Psychoneuroendocrinology 23:353-370.

Spangler G. 1997. Psychological and physiological responses during an exam and their relation to personality characteristics. Psychoneuroendocrinology 22(6):423-441.

Spielberger CD. 1983. State-trait anxiety inventory. Palo Alto: Mind Garden.

Vedhara K, Hyde J, Gilchrist ID, Tytherleigh M, Plummer S. 2000. Acute stress, memory, attention and cortisol. Psychoneuroendocrinology 25:535-549.

Wachtel SR, Charnot A, de Wit H. 2001. Acute hydrocortisone administration does not affect subjective responses to Damphetamine in humans. Psychopharmacology 153:380-388.

Wechsler D. 1981. Manual for the Wechsler adult intelligence scale-revised. New York: The Psychological Corporation.

Wechsler D. 1987. Manual for the Wechsler memory scale - revised. San Antonio: The Psychological Corporation.

Weekes NY, Lewis RS, Patel FR, Garrison-Jakel J, Berger D, Lupien SJ. 2006. Validation of examination stress as an ecological inducer of cortisol and psychology responses to stress controlling for sex, time of day and seasonal effects. Stress 9:199-206.

Wolf OT, Convit A, McHugh PF, Kandil E, Thorn EL, De Santi S, de Leon MJ. 2001. Cortisol differentially affects memory in young and elderly men. Behav Neurosci 115:1002-1011.

Yerkes RM, Dodson JD. 1908. The relation of strength of stimulus to rapidity of habit-formation. J Comp Neurol Psychol 18: $459-482$.

Young AH, Sahakian BJ, Robbins TW, Cowen PJ. 1999. The effects of chronic administration of hydrocortisone on cognitive function in normal male volunteers. Psychopharmacology 145:260-266. 
Copyright of Stress: The International Journal on the Biology of Stress is the property of Taylor \& Francis Ltd and its content may not be copied or emailed to multiple sites or posted to a listserv without the copyright holder's express written permission. However, users may print, download, or email articles for individual use. 
Copyright of Stress: The International Journal on the Biology of Stress is the property of Taylor \& Francis Ltd and its content may not be copied or emailed to multiple sites or posted to a listserv without the copyright holder's express written permission. However, users may print, download, or email articles for individual use. 